\title{
Design of a High Contrast Grating GaSb-based VCSEL integrated on Silicon-On-Insulator
}

\author{
R.Wang, D.Sanchez and G.Roelkens \\ Photonics Research Group, INTEC Department, Ghent University-IMEC, Sint-Pietersnieuwstraat 41, 9000 Ghent, Belgium
}

\begin{abstract}
We present a GaSb-VCSEL concept using SOI-based HCGs as highly reflective mirrors. The optical properties of two different grating designs are simulated using RCWA. These gratings show strong and broadband reflection around $2.3 \mu \mathrm{m}$.
\end{abstract}

\section{INTRODUCTION}

The mid-infrared (MIR) wavelength range and especially the $2-3.5 \mu \mathrm{m}$ range is of particular interest for spectroscopic sensing applications. The presence of different polluting species such as methane, ammonia or carbon monoxide, can be easily detected using a technique called Tunable Diode Laser Absorption Spectroscopy (TDLAS), which requires mode-hop free tuning and single-mode laser emission [1]. Electrically-Pumped GaSb-based Vertical Cavity Surface Emitting Lasers (EP-VCSELs) are good candidates for this application. Recently, EP-VCSELs emitting at wavelengths above $2 \mu \mathrm{m}$ have been demonstrated; these are monolithic [2] or hybrid [3] devices with low-threshold current and single-mode emission. The current injection is made through one or two semiconductor Distributed Bragg Reflectors (DBRs), which introduce excessive resistive heating and optical losses into the structure. Replacing these semiconductors DBRs by a High-Contrast Grating (HCG) mirror is therefore very promising, especially since it permits a reduction of the structure thickness, provides polarization control and allows large aperture single-transverse mode emission [4]. HCGs have been studied over the last years and VCSELs using HCGs instead of DBRs have been demonstrated recently in the GaAs [4] and InP [5-6] system.

VCSELs integrated on SOI emitting in the midinfrared have not been presented up to now. In this paper, we introduce the concept of GaSb-based EPVCSELs BCB-bonded on SOI using an HCG as outcoupling mirror. We study $\mathrm{SiO} 2 / \mathrm{Si}$ and air/Si HCGs for a high reflectivity around $2.3 \mu \mathrm{m}$ and study the properties of these HCGs using RCWA and FDTD.

\section{VCSEL DESIGN}

The EP-VCSEL concept using a HCG as an outcoupling mirror is presented by the scheme in Figure 1-a. The structure is based on a half epitaxial VCSEL grown on a $\mathrm{n}-\mathrm{GaSb}$ substrate by Molecular Beam Epitaxy. The Sb-based epitaxial stack is then BCBbonded onto a SOI die where the HCGs were preprocessed. Substrate removal and post-bonding processing is then applied to the Sb-epitaxial stack. Post-bonding processing of the VCSEL allows bonding the two wafers without stringent alignment since the mesa definition, the electro-optical confinement by selective etching of the InAsSb/GaSb tunnel-junction [2] and the contact deposition are done after bonding, lithographically aligned to the silicon structures.

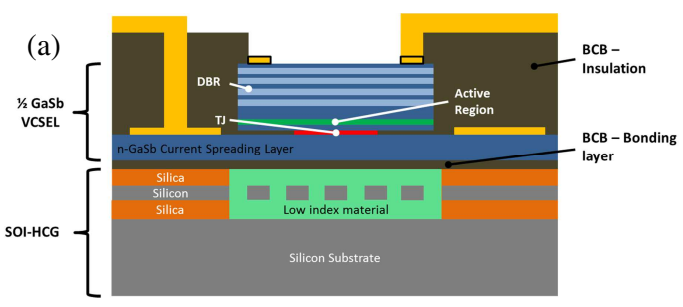

(b)



Figure 1: (a) Scheme of the BCB-bonded Sb-based EPVCSEL using a HCG as out-coupling mirror. (b) Scheme of the HCG reflector, the $220 \mathrm{~nm}$-thick SOI is surrounded by lower index material (air or $\mathrm{SiO} 2$ ).

Figure 1-b presents a schematic of the HCG used as out-coupling mirror $(\mathrm{R}>99 \%)$. Since the fabrication process is based on a Silicon-On-Insulator (SOI) platform process, the silicon layer and buried oxide thickness are fixed to 220 and $2000 \mathrm{~nm}$ respectively. The SOI platform provides high index material such as $\mathrm{Si} \quad(\mathrm{n} \approx 3.45)$ and low index material like $\mathrm{SiO} 2$ $(\mathrm{n} \approx 1.45)$, but also air $(\mathrm{n} \approx 1)$. HCGs made of these materials provide good reflection characteristics. Varying the grating period $(\Gamma)$ and Fill Factor $(F F)$ of the grating, one can modify the reflectivity and phase of the reflector. The first design is based on a $220 \mathrm{~nm}$ thick silicon layer surrounded by silicon dioxide as a low index material, which requires no further processing after III-V processing. It should allow processing arrays of VCSELs emitting at different wavelengths by bonding on HCGs with different periods and FF parameters. The second design is based on free-standing silicon gratings, which require additional technological steps in order to wet etch the silicon dioxide surrounding the silicon after the fabrication process. This design has however the 
advantage of allowing electro-static actuation, which allows modifying the cavity length and hence the lasing wavelength by applying a voltage between the silicon membrane and the silicon substrate or III-V.

\section{RESULTS}

The reflectivity spectra for a plane wave with its Efields parallel (TE) to the Si-HCG are calculated using rigorous coupled wave analysis (RCWA) and FDTD as plotted in Figure 2. The high-reflection spectrum $\left(\mathrm{R}_{0}>99 \%\right)$ exhibits a bandwidth of $73 \mathrm{~nm}$ and $186 \mathrm{~nm}$ for oxide $(\mathrm{Gamma}=1.27 \mu \mathrm{m}, \mathrm{FF}=55 \%)$ and air cladding HCGs $(\mathrm{Gamma}=1.5 \mu \mathrm{m}, \mathrm{FF}=50 \%)$ respectively.

For a normal incident plane wave, Si-HCGs have broadband high reflectivity as shown in Figure 2. But the beam profile in VCSELs usually carries higher incident angle components, which are determined by the optical aperture.
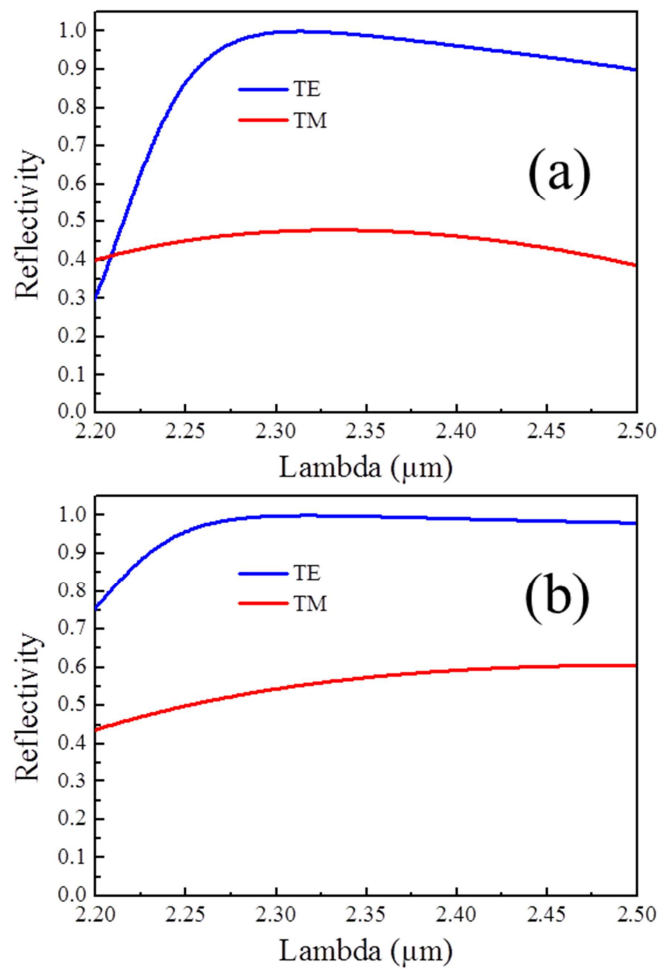

Figure 2: Reflectivity spectra for surface-normal inciden plane waves on an oxide cladding HCG (a) and an air cladding HCG (b).

The angle dependence of the two designs is also investigated (not shown there). The oxide cladding HCG can tolerate an incident angle variation from zero degree to \pm 5 degrees at a wavelength of $2.3 \mu \mathrm{m}$, for the air cladding Si-HCG, the angular tolerance is around \pm 10 degrees. The reflectance of the aircladding HCG is still higher than $99 \%$ above \pm 7 degrees at the wavelength of $2.36 \mu \mathrm{m}$, showing the great potential of such a design in a widely tunable VCSEL.
In order to confirm the obtained results for infinite gratings illuminated by a plane wave, twodimensional FDTD simulations were carried out to obtain the cavity Q-factor of the hybrid $\mathrm{GaSb}$ on $\mathrm{Si}$ VCSEL structure. This is shown in Figure 4, illustrating the cavity mode field distribution and the resonance peak of the cavity, yielding a Q-factor of around 950. The inset also shows how the resonance wavelength and the Q-factor of the cavity mode evolve when the grating is actuated.
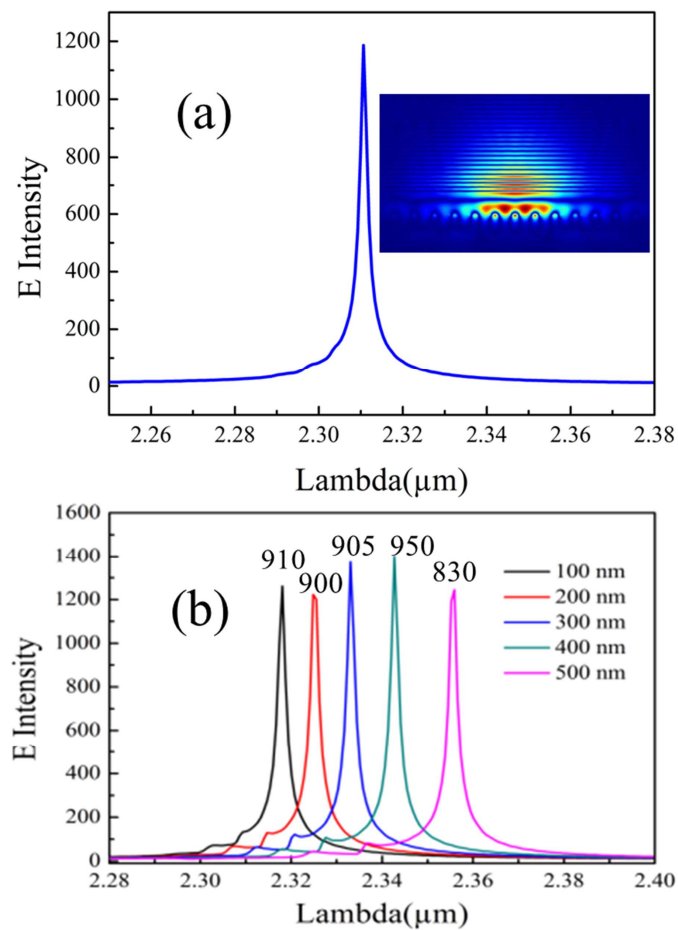

Figure 3: VCSEL mode profile and cavity Q-factor for the air-clad VCSEL structure.

\section{REFERENCES}

[1] Vicet et al., "Trace gas detection with antimonide-based quantum-well diode lasers" Spectrochimica acta. Part A, vol. 58 , no. $11,2002$.

[2] Sanchez et al., "Single-Mode Monolithic GaSb VerticalCavity Surface-Emitting Laser" Optics Express, vol. 20, no. 14, 2012.

[3] Bachmann et al., "Long wavelength electrically pumped GaSb-based buried tunnel junction VCSELs" Physics Procedia, vol. 3, no. 2, 2010.

[4] Huang et al., "A surface-emitting laser incorporating a high-index-contrast subwavelength grating" Nature Photonics, vol. 1, no. 2, 2007.

[5] Chase et al., "1550 nm high contrast grating VCSEL" Optics express, vol. 18, no. 15, 2010.

[6] Sciancalepore et al., "CMOS-compatible ultra-compact $1.55-\mu \mathrm{m}$ emitting VCSELs using double photonic crystal mirrors" Photonics Technology letters, vol. 24, no. 6, 2012. 